\title{
Estimation of the characteristic energy of electron precipitation
}

\author{
C. F. del Pozo, M. J. Kosch, and F. Honary \\ Department Communication Systems, Lancaster University, Lancaster LA1 4YR, UK
}

Received: 15 October 2001 - Revised: 19 July 2002 - Accepted: 24 August 2002

\begin{abstract}
Data from simultaneous observations (on 13 February 1996, 9 November 1998, and 12 February 1999) with the IRIS, DASI and EISCAT systems are employed in the study of the energy distribution of the electron precipitation during substorm activity. The estimation of the characteristic energy of the electron precipitation over the common field of view of IRIS and DASI is discussed. In particular, we look closely at the physical basis of the correspondence between the characteristic energy, the flux-averaged energy, as defined below, and the logarithm of the ratio of the greenlight intensity to the square of absorption. This study expands and corrects results presented in the paper by Kosch et al. (2001). It is noticed, moreover, that acceleration associated with diffusion processes in the magnetosphere long before precipitation may be controlling the shape of the energy spectrum. We propose and test a "mixed" distribution for the energy-flux spectrum, exponential at the lower energies and Maxwellian or modified power-law at the higher energies, with a threshold energy separating these two regimes. The energy-flux spectrum at Troms $\varnothing$, in the $1-320 \mathrm{keV}$ range, is derived from EISCAT electron density profiles in the 70$140 \mathrm{~km}$ altitude range and is applied in the "calibration" of the optical intensity and absorption distributions, in order to extrapolate the flux and characteristic energy maps.
\end{abstract}

Key words. Ionosphere (auroral ionosphere; particle precipitation; particle acceleration)

\section{Introduction}

At high latitudes, precipitating energetic particles collide with the neutral constituents in the atmosphere and generate light emission and ionisation in the D- and E-regions and act as "tracers" in a number of solar-terrestrial processes. In the auroral region, most events are due to the precipitation of energetic electrons during various levels of magnetic dis-

Correspondence to: C. F. del Pozo

(c.del.pozo@lancaster.ac.uk) turbance and substorm activity. The size, structure, and evolution of both the absorption and the optical signatures are measures of the properties of the ionisation patches and corresponding optical forms, as well as of the associated precipitation source in the magnetosphere. Many observations have established that fluctuations in auroral intensity are correlated with variations in absorption, as well as with the ion production and average electron densities at the corresponding height ranges where most of the optical emission and absorption occur (Rees, 1963; Johansen, 1965; Berkey, 1968; Gustafsson, 1969; Stoker et al., 1996). (See the book by Rees, 1989 for a thorough review of the theoretical and experimental basis underlying these correlations.)

The aim of this study is to correct and expand the initial results from the paper by Kosch et al. (2001) and to present some physical arguments to justify the method to estimate the characteristic energy and flux of the precipitating electrons over the common field of view of the IRIS and DASI instruments. In particular, we look closely at the physical basis of the observed correspondence between the characteristic energy defined below, and the logarithm of the ratio of the green-light intensity to the square of absorption. We also include new data and look in some detail at the estimation of the characteristic energy and the flux-averaged energy of the energy-flux spectrum.

Kosch et al. (2001) have demonstrated that it is possible to produce a two-dimensional map of the characteristic energy of precipitating particles for a given spectral function (e.g. Maxwellian or exponential). Assuming that the high energy $(>25 \mathrm{keV})$ and low energy $(<15 \mathrm{keV})$ components of the particle precipitation come from the same population in the magnetosphere, with D-region radio-wave absorption (Abs) proportional to the square root of the flux of high energy particles $(>25 \mathrm{keV})$ and E-region auroral intensity at $557.7 \mathrm{~nm}$ (Int) proportional to the flux of low energy particles $(<15 \mathrm{keV})$, the characteristic energy $(E o)$ of an assumed energy distribution is proportional to the logarithm of ratio $\mathrm{Int} /(\mathrm{Abs})^{2}$. Kosch et al. (2001), however, made a mistake by comparing the characteristic energy $E o$ with $\log \left(\operatorname{Int}^{0.5} / \mathrm{Abs}\right)$. 
This mistake did indeed propagate to their results, but fortunately, because the characteristic energy $E o$ is proportional to $\log \left(\operatorname{Int} / \mathrm{Abs}^{2}\right)$, and not to $\log \left(\mathrm{Int}^{0.5} / \mathrm{Abs}\right)$, as they incorrectly stated, the structure of their "energy maps" was not affected but only the energy levels by a linear factor of the order of 2 (allowing for some dispersion due to the statistical nature of the linear regression between $\log \left(\mathrm{Int} / \mathrm{Abs}^{2}\right)$ and $\log$ $\left(\mathrm{F}\left(\mathrm{E}_{2}\right) / \mathrm{F}\left(\mathrm{E}_{1}\right)\right)$.

The energy-flux spectrum of electrons at Troms $\varnothing$, in the $1-320 \mathrm{keV}$ range, is derived from electron density profiles measured with EISCAT UHF radar in the $70-140 \mathrm{~km}$ altitude range (del Pozo et al., 1997, 1999) and applied in the "calibration" of the green-line intensity and absorption distributions, in order to extrapolate the flux and characteristic energy maps over the common field of view of the IRIS and DASI systems. EISCAT (European Incoherent Scatter at $933 \mathrm{MHz}$, Rishbeth and Williams, 1985) is a tri-static system with fully steerable antennas $32-\mathrm{m}$ in diameter at Troms $\varnothing$ $\left(69.6^{\circ} \mathrm{N}, 19.2^{\circ} \mathrm{E}\right)$, Kiruna $\left(67.9^{\circ} \mathrm{N}, 20.4^{\circ} \mathrm{E}\right)$ and Sodankylä $\left(67.4^{\circ} \mathrm{N}, 26.7^{\circ} \mathrm{E}\right)$. It measures the height profiles of various ionospheric parameters $(\mathrm{Ne}, \mathrm{Te}, \mathrm{Ti})$ and the electric field.

IRIS (Imaging Riometer for Ionospheric Studies) at Kilspisjärvi $\left(69.05^{\circ} \mathrm{N}, 20.8^{\circ} \mathrm{E}\right)$ records the absorption of cosmic radio noise at $38 \mathrm{MHz}$ as measured in 49 different directions (Browne et al., 1995). The bulk of the absorption corresponds to ionisation caused by electrons with initial energies ranging from a few $10 \mathrm{keV}$ up to a few $100 \mathrm{keV}$, with deposition heights generally centred at $\sim 90 \mathrm{~km}$. IRIS gives a picture of the horizontal extent of the ionisation with a minimum time resolution of $1 \mathrm{~s}$. Spatial resolution, as defined by the 3-dB points of the beams, ranges between 20 and $60 \mathrm{~km}$ (Collis et al., 1996). DASI (Digital All Sky Imager, (Kosch et al., 1998), at $69.35^{\circ} \mathrm{N}, 20.36^{\circ} \mathrm{E}$ ) measures the green-light auroral emission in rayleighs at $557.7 \mathrm{~nm}$ at $10 \mathrm{~s}$ resolution. The zenith angle of the original all-sky images is converted to north-south horizontal distance from vertical (near Tromsø), with an overall spatial resolution of $10 \times 10 \mathrm{~km}$, although the absolute resolution is $0.1^{\circ}$ in zenith angle, which goes from $200 \mathrm{~m}$ to $4 \mathrm{~km}$ for zenith angles from $0^{\circ}$ to $80^{\circ}$. All-sky cameras record the light emission from the excitation of neutral gases and ions by precipitating electrons with energies between $100 \mathrm{eV}$ and a few $\mathrm{keV}$. Most of the auroral luminosity is due to the green-light emission from the altitude range $90-120 \mathrm{~km}$, with peak frequency of occurrence between 100 and $110 \mathrm{~km}$ (Egeland and Omholt, 1967). The effective IRIS field of view at $90-100 \mathrm{~km}$ height is a square of $240-\mathrm{km}$ side centred at Kilpisjärvi and corresponding to $6^{\circ}$ lon. (17.75 $23.75^{\circ}$ lon.) and $2.4^{\circ}$ lat. ( $67.8^{\circ}-70.2^{\circ}$ lat.). The standard digitised coverage from DASI is a square of $500 \mathrm{~km}$ side centred at Skibotn, or $12.5^{\circ}$ lon. $\left(13.5^{\circ}-26^{\circ}\right.$ lon.) and $5^{\circ}$ lat. $\left(67.6^{\circ}-72.6^{\circ}\right.$ lat. $)$.

\subsection{Characteristic energy, absorption and luminosity}

The flux of precipitating electrons can be represented by a modified power-law dependence, with the exponential and
Maxwellian spectra as particular cases (Rees, 1989):

$F(E)=A o(E / E o)^{\gamma} \exp (-E / E o)$,

with $\gamma=0$ for an exponential, 1 for a Maxwellian, and $\gamma \geq 2$, for a modified power-law spectrum. Actually, both the exponential and Maxwellian spectra come from Maxwellian velocity distributions. The plain exponential comes from an uni-directional Maxwellian distribution for particles with very narrow pitch-angle dispersion, while the so-called Maxwellian spectrum is associated with an isotropic Maxwellian distribution. Maxwellian and modified power law spectra are suggestive of an electron population that has been accelerated long before precipitation takes place. The modified power-law spectrum is not the direct output of a physical process, but a representation of the simultaneous stretching and widening of the tail of the Maxwellian distribution during the acceleration processes. On the other hand, particle acceleration along the field lines takes place after precipitation and is signalled by the presence of bumps in the spectrum and associated with drifting Maxwellians (Rostoker et al., 1985).

$E o$ is the characteristic energy and gives the energy of the precipitating electrons at the top of the ionosphere. The characteristic energy can be determined from the flux at two energy bands $E_{1}>E_{2}$

$E o=\left(E_{1}-E_{2}\right) \log \left[\left(E_{1} / E_{2}\right)^{\gamma} F\left(E_{2}\right) / F\left(E_{1}\right)\right]$.

If the spectrum is represented by a distribution from Eq. (1), then the flux-averaged energy, $\langle E\rangle$, gives a good estimate of the characteristic energy when integrating over the full spectrum or between energy bands $E_{A}$ and $E_{B}$, such that $E_{B} \gg E o \gg E_{A}$ :

$\langle E\rangle=\Sigma_{i} E_{i} F\left(E_{i}\right) / \Sigma_{k} F\left(E_{k}\right) \sim(\gamma+1) E o$.

We may define a "mixed" spectrum, approaching an exponential at the lower energies and a modified power-law distribution at the higher energies, with some kind of energy "threshold", $E_{C}(>E o)$, separating these two regimes:

$$
\begin{aligned}
& \mathrm{F}(\mathrm{E})=\text { Ao } \exp (-\mathrm{E} / \mathrm{Eo}) \text { for } \mathrm{E}<\mathrm{E}_{\mathrm{C}}, \\
& F(E)=A o\left(E / E_{C}\right)^{\gamma} \exp (-E / E o) \text { for } E>E_{C} .
\end{aligned}
$$

Energy $E_{C}$ may be a function of $E o$, the pitch angle, etc., and may even change with time and the geomagnetic disturbance level. In any case, for this mixed spectrum, we also have that $\langle E\rangle \sim E o$, because the determination of the total flux and the flux-averaged energy are dominated by the exponential side of the spectrum. We will see in Sect. 3 that most of the estimated flux in our observations is concentrated at the lower energies where is closely represented by an exponential distribution. At the higher energies, on the other hand, we have more flux than in the full exponential spectrum, and the spectral shape may be better approximated by the Maxwellian or the modified power-law distributions. This means that a mixed distribution (or rather a smoother 
IRIS/DASI Keograms (at Long. 19.25 $\mathrm{E}$ )

(a) 13-14 Feb. 96, (b) 09 Nov. 98, (c) 12 Feb. 99
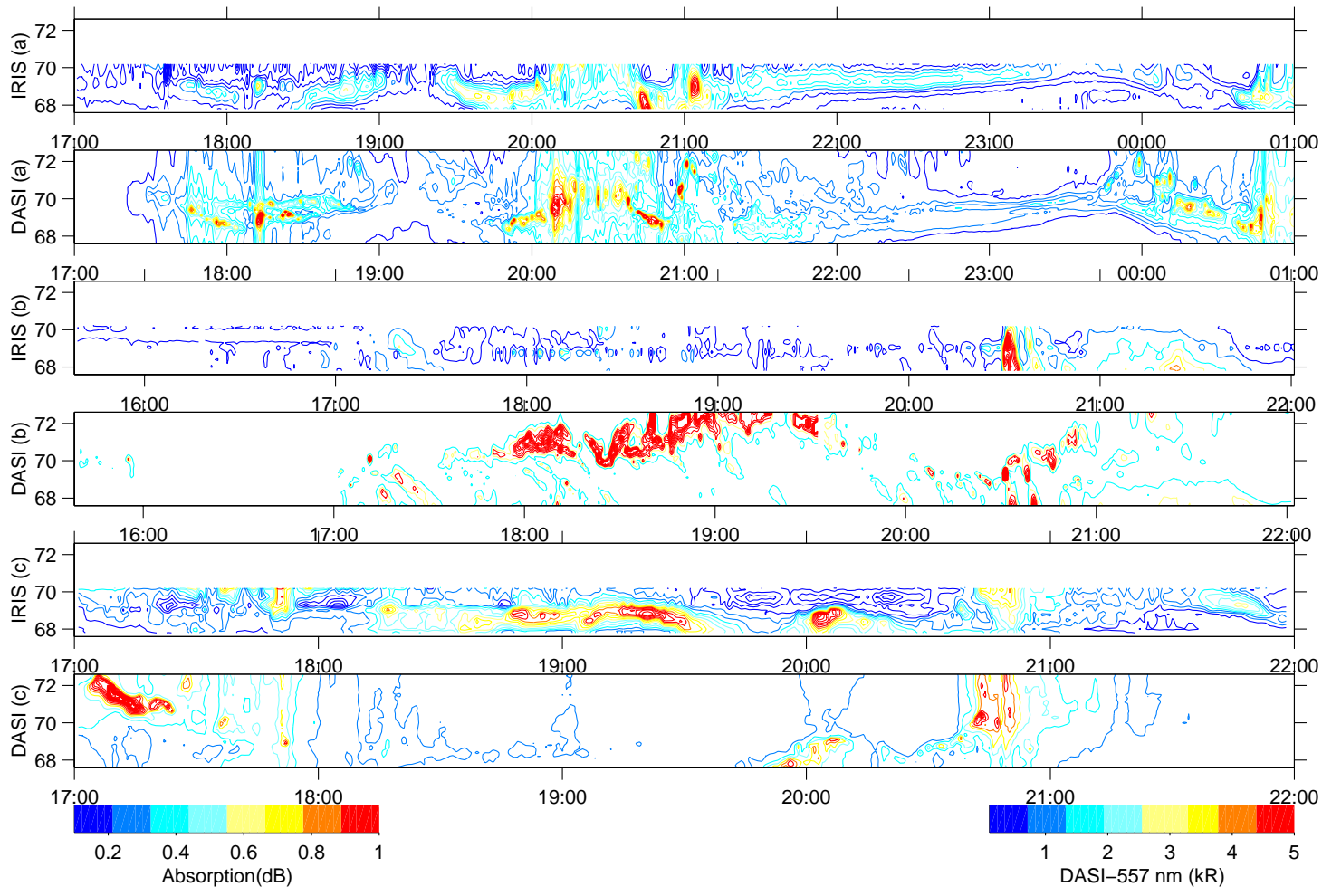

Fig. 1. Keograms at $1 \mathrm{~min}$ resolution from IRIS absorption and from DASI green-light intensity for observation periods: (a) 17:00-01:00 UT, 13-14 Feb 1996, (b) 15:30-22:00 UT, 09 Nov 1998, and (c) 17:00-22:00 UT, 12 Feb 1999. Colour bars are given on the bottom in dB for the absorption and in $\mathrm{kR}$ for the optical emission.

version of Eq. 4) may give a better representation of the estimated flux spectrum.

At the flux-averaged deposition energies $E_{1}, E_{2}$ in ranges $85-95 \mathrm{~km}$ and $108-120 \mathrm{~km}$ :

$\operatorname{Abs}(t)^{2} \sim K_{1} F\left(E_{1}, t\right)$,

$I_{\lambda, X}(t) \sim K_{2} F\left(E_{2}, t\right)$,

where $\operatorname{Abs}(t)$ the total (height-integrated) absorption is given by Hargreaves (1992) and $I_{\lambda, X}(t)$ is the total luminosity or height-integrated emission due to the precipitating electrons, at a given wavelength and for atmospheric constituent $X(557.7 \mathrm{~nm}$ and oxygen in this case) (see, for instance, Rees, 1963; Oksman, 1996). The proportionality factors $K_{1}$ and $K_{2}$ may change at time scales much larger than the ion recombination time and, therefore, very slowly compared to the rapid changes in particle flux and characteristic energy. At the shorter time scales, changes in electron density are directly related to changes in the precipitation flux. These last two equations correct the derivations presented by Kosch et al. (2001; their Eqs. 10 and 11). Actually, the absorption (Abs) is basically proportional to the electron density at a relatively narrow height range centred at $z_{A}\left[\operatorname{Abs}(t) \propto N e\left(z_{A}, t\right)\right]$, and for corresponding deposition energy $E_{A}$ of the precipitating electrons. At these heights, the ion production rate is proportional to the square of the electron density, and the production rate is in its turn, proportional to the precipitation flux $F\left(E_{A}\right)$ (or, equivalently, $F\left(E_{A}, t\right) \propto Q\left(z_{A}, t\right) \sim \alpha_{\mathrm{eff}}\left(z_{A}\right) N e\left(z_{A}, t\right)^{2}, \alpha_{\mathrm{eff}}(z)$ is the effective ion recombination rate). The optical intensity (Int) is, on the other hand, basically proportional to the production rate at also a relatively narrow height range centred at $z_{I}$ with corresponding energy $E_{I}$ (then, $\operatorname{Int}\left(z_{I}, t\right) \propto Q\left(z_{I}, t\right) \propto$ $\left.F\left(E_{I}, t\right)\right)$.

\section{Experimental data}

The data discussed in this study were obtained during three periods of simultaneous winter nighttime observations with the EISCAT, IRIS and DASI systems:

(1) 17:00-01:00 UT on 13-14 February 1996,

(2) 15:30-22:00 UT on 9 November 1998, and 


\section{EISCAT DATA - ESTIMATED FLUX SPECTRUM 12/02/99 - 17:00:00 TO 12/02/99 - 21:51:00 UT}

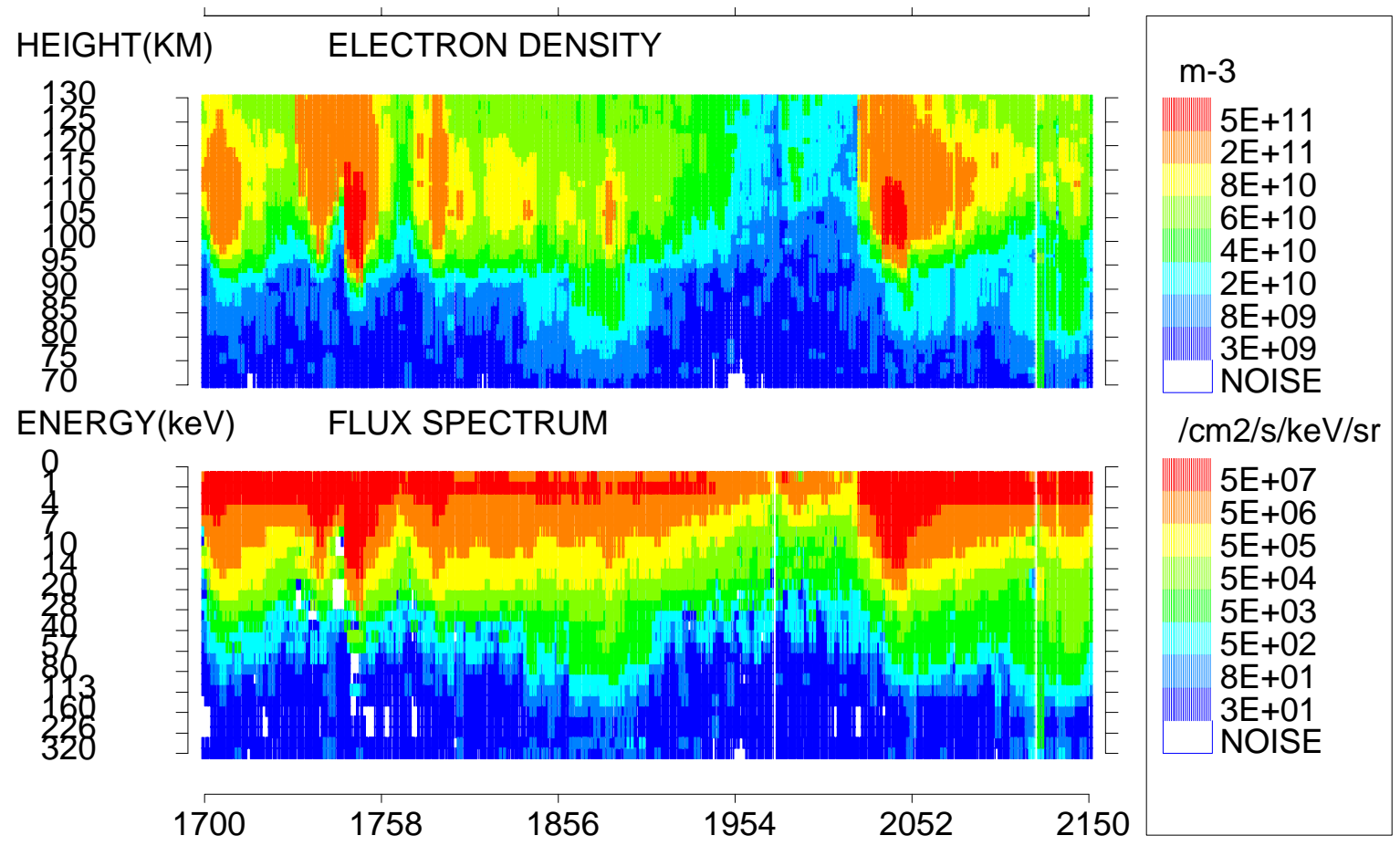

UNIVERSAL TIME

Fig. 2. (Top) Electron density profiles measured by EISCAT and (bottom) calculated energy-flux spectra (the differential flux) during 12 Feb 1999 at 17:00-22:00 UT and 1 min resolution. The colour scales are given on the right-hand side in $\mathrm{m}^{-3}$ for the electron density and in $/ \mathrm{keV} / \mathrm{cm}^{2} / \mathrm{s} / \mathrm{sr}$ for the flux.

\section{(3) 17:00-22:00 UT on 12 February 1999.}

In all cases, the three instruments recorded the occurrence of multiple substorm onsets and the presence of auroral forms and arcs moving across their respective fields of view, consistent with the drift patterns at substorm growth, expansion and recovery phases (particularly in period 1). We have considered an initial time resolution of $10 \mathrm{~s}$ for EISCAT, IRIS and DASI data, and then we post-integrate to a 1 min. resolution. In all cases, the EISCAT UHF antenna was kept pointing along the magnetic field line at Troms $\varnothing$. The inversion algorithm to estimate the energy-flux spectrum from the radar observations was applied at these two resolutions. However, given the large amount of data points and the various types of calculations performed, we restrict our analysis to a common resolution of $1 \mathrm{~min}$ for all data sets.

Figure 1 shows the summary plots of the IRIS and DASI data for these three observation periods. It displays together the keograms at $19.25^{\circ}$ lon. ( $\sim$ Troms $\varnothing$ ) of absorption and optical intensity (at $557.7 \mathrm{~nm}$ ). Colour bars on the bottom give the absorption in $\mathrm{dB}$ (to the left) and the green-light intensity in kRayleighs ( $\mathrm{kR}$, to the right). The close coincidence between maximum intensities indicates the presence of sub- storm activity within the common field of view, and this is particularly true on 13-14 February 1996, and to a lesser extent on 12 February 1998. In the other cases, the optical activity was more intense to the north, such as during 9 November 1998. The brightest aurora occurred during 9 November 1998 , with maximum green-line intensity of $32 \mathrm{kR}$, but because it fell mostly outside the IRIS field of view, the absorption is low, particularly before 20:00 UT. The maximum intensity at the longitude of Troms $\varnothing$ during 13-14 February 1996 was 7 kR, and during 12 February 1999 was 16 kR.

Figure 2, on the other hand, shows the raw electron density measured by EISCAT in the $70-130 \mathrm{~km}$ range and the corresponding electron precipitation flux (the differential flux, per $\mathrm{keV}$, per $\mathrm{cm}^{2}$, per second and per ster-radian) from the inversion of the electron density profiles for the observing period 12 February 1999, 17:00-21:51 UT. The time resolution is $1 \mathrm{~min}$ in both cases, and the colour-coded scales are displayed on the right-hand side. The reader is referred to the paper by Kosch et al. (2001) for similar plots presenting the data for the 13-14 February 1996 and 9 November 1998 periods. Meaningful determinations of the flux spectrum were only possible for electron densities greater than $5 \times 10^{9} \mathrm{~m}^{-3}$ at any given height (see, del Pozo et al., 1997). This was 
roughly the sensitivity limit for the EISCAT UHF determinations before the very recent system refurbishment. As you may see from the figure, the electron densities are above this value for heights greater than $75 \mathrm{~km}$ and for corresponding flux-spectrum energies smaller than $200 \mathrm{keV}$. The energyflux spectrum is estimated by the inversion of the electron density profile in the height range $70-140 \mathrm{~km}$, over 28 energy bands in the range $1-320 \mathrm{keV}$, the effective deposition height, $h$, for precipitating electrons can be related to their energy by $E(h)=4 \times 10^{5} \exp (-0.101 \mathrm{~h})$ (see, del Pozo et al., 1997; Kosch et al., 2001).

\section{Discussion}

The results and the heuristic arguments presented in Kosch et al. (2001) and in this study show the presence of a generally good linear correlation between the particle fluxes and the optical emission and absorption, at the two corresponding height ranges and energy bands for the precipitating electrons. This correlation also justifies the relatively good linear regression between the logarithm of the ratio of the average fluxes, at the two height ranges, and the logarithm of the ratio of the optical intensity to the absorption measured at Troms $\varnothing$, and it allows the estimation of the characteristic energy of the precipitating electrons. Kosch et al. (2001) simply combined the 13 February 1996 and 9 November 1998 data sets. Here we analyze the individual data sets (including the additional period in 12 February 1999) and then all together to see whether determining $E o$ from the absorption and optical intensity is consistent over time. We are also investigating if a physical parameter, such as the flux-averaged energy directly derived from the EISCAT measurements - can be employed as a reference for, and to further qualify, our determinations of the characteristic energy and help us decide which type of spectral distribution (given by Eq. 1 or Eq. 4) may better represent the energy-flux spectrum from the measurements.

Figure 3 presents the dispersion plots for the fluxes, absorption and optical intensity for the three data sets (frames (a), (b), and (c)) at Troms $\varnothing$; the choice of a log-log scale is only a matter of convenience. The $y$-axis displays, in the upper panels the $\log$ of the square of absorption and in the bottom panels the logarithm of the $557.7 \mathrm{~nm}$ intensity. The $x$-axis displays, in all cases, the logarithm of the electron flux at the corresponding height/energy ranges. Flux 1 and flux 2 are averages over the height ranges $108-120 \mathrm{~km}$ and $85-95 \mathrm{~km}$, respectively, with corresponding energy ranges 2$7 \mathrm{keV}$ and $27-75 \mathrm{keV}$. The calculated correlation coefficients, from Fig. 3, between Flux $F_{1}$ and $\mathrm{Abs}^{2}$, and between Flux $F_{2}$ and Int (the green-light intensity) are: 0.64 and 0.51 (with 480 data points) for the 13-14 February 1996 data, 0.72 and 0.80 (with 300 data points) for the 9 November 1998 data, and 0.34 and 0.86 (with 300 data points) for the 12 February 1999 data. Given the large size of the samples in all three periods, the actual populations' correlation coefficient are indeed greater than their sample estimates.
Figure 4 gives the dispersion plots between the logarithm of the ratio of the optical intensity to the square of the absorption and the logarithm of the ratio of the corresponding fluxes. Frames (a), (b), and (c) correspond to the same data sets as Fig. 3, and frame (d) displays all the data points together. Remember that for spectral distributions of the type given in Eq. (1) (and in Eq. (4) as well), the characteristic energy is inversely proportional to the log of the ratio $F_{2} / F_{1}$ (Eq. 2). The regression lines are shown in red on the dispersion plots, the dotted lines on both sides and in all cases give one standard deviation intervals for the linear regression. The regression line is given by:

$\log \left(\operatorname{Int} / \mathrm{Abs}^{2}\right) \sim a+b \log \left(F_{2} / F_{1}\right)$.

Flux $F_{1}$ in energy band $E_{1}$ is responsible for most of the absorption (Abs) recorded by IRIS, while flux $F_{2}$ in the band $E_{2}$ causes most of the green-light emission (Int). $E_{1}$ and $E_{2}$ are, in fact, the flux-averaged deposition energies over the height ranges $85-95 \mathrm{~km}$ and $108-120 \mathrm{~km}$, respectively, and equal to $37.5 \mathrm{keV}$ and $2.72 \mathrm{keV}$ in 13-14 February 1996 ; equal to $33.6 \mathrm{keV}$ and $2.85 \mathrm{keV}$ in 9 November 1998 ; and equal to $38.9 \mathrm{keV}$ and $3.05 \mathrm{keV}$ in 12 February 1999 . We are considering fixed values $E_{1}=40 \mathrm{keV}$ and $E_{2}=3 \mathrm{keV}$ in all our calculations, and thus assuming that the energy/height range at which most of the absorption and green-line emission takes place changes little during the length of the observation periods. This assumption seems justified a posteriori by the good correlation between these parameters and the EISCAT electron density profiles or, equivalently, the electron fluxes at the corresponding height ranges. Moreover, due to the significant linear correlation between $F_{1}$ and $\mathrm{Abs}^{2}$, and $F_{2}$ and Int, we could expect a similar dependence between the $E o$ and $\log \left(\operatorname{Int} / \mathrm{Abs}^{2}\right)$, in the case where the shape of the spectrum between energies $E_{1}$ and $E_{2}$ can be approximated by either an exponential, a Maxwellian or a modified power-law distribution, or by the "mixed" distribution in Eq. (4). We may have,

$\log \left(\mathrm{Int} / \mathrm{Abs}^{2}\right) \sim a^{\prime}+b\left(E_{1}-E_{2}\right) / E o$.

The value of $a^{\prime}$ is obtained from, $a$, in Eq. (7) by $a^{\prime}=$ $a-b \log \left(E_{1} / E_{2}\right)^{\gamma}$ or, for a mixed distribution, $a^{\prime}=a-$ $b \log \left(E_{1} / E_{C}\right)^{\gamma}$. In this paper we have assumed a fixed value of $E_{C}=25 \mathrm{keV}$, considering the fact that up to $20 \mathrm{keV}$ the shape of the flux spectrum may be reasonably represented by an exponential distribution (see Fig. 5a). Moreover, optical emissions occur above $100 \mathrm{~km}$ and result from precipitating electrons with energies smaller than $15 \mathrm{keV}$, and riometer absorption normally occurs below $95 \mathrm{~km}$ for energies greater than $25 \mathrm{keV}$.

Parameters $\mathrm{a}$ and $\mathrm{b}$ from the linear regressions are: $a=$ $7.24, b=0.33$ for $13-14$ February $1996, a=7.57, b=0.45$ for 9 November 1998, $a=6.97, b=0.34$ for 12 February 1999 and, for all data points together, $a=7.71, b=0.29$. In all cases, most points fall within one standard deviation from the regression line with a $99 \%$ confidence interval. Notice that $E o$ is proportional to the logarithm of the fluxes and to 

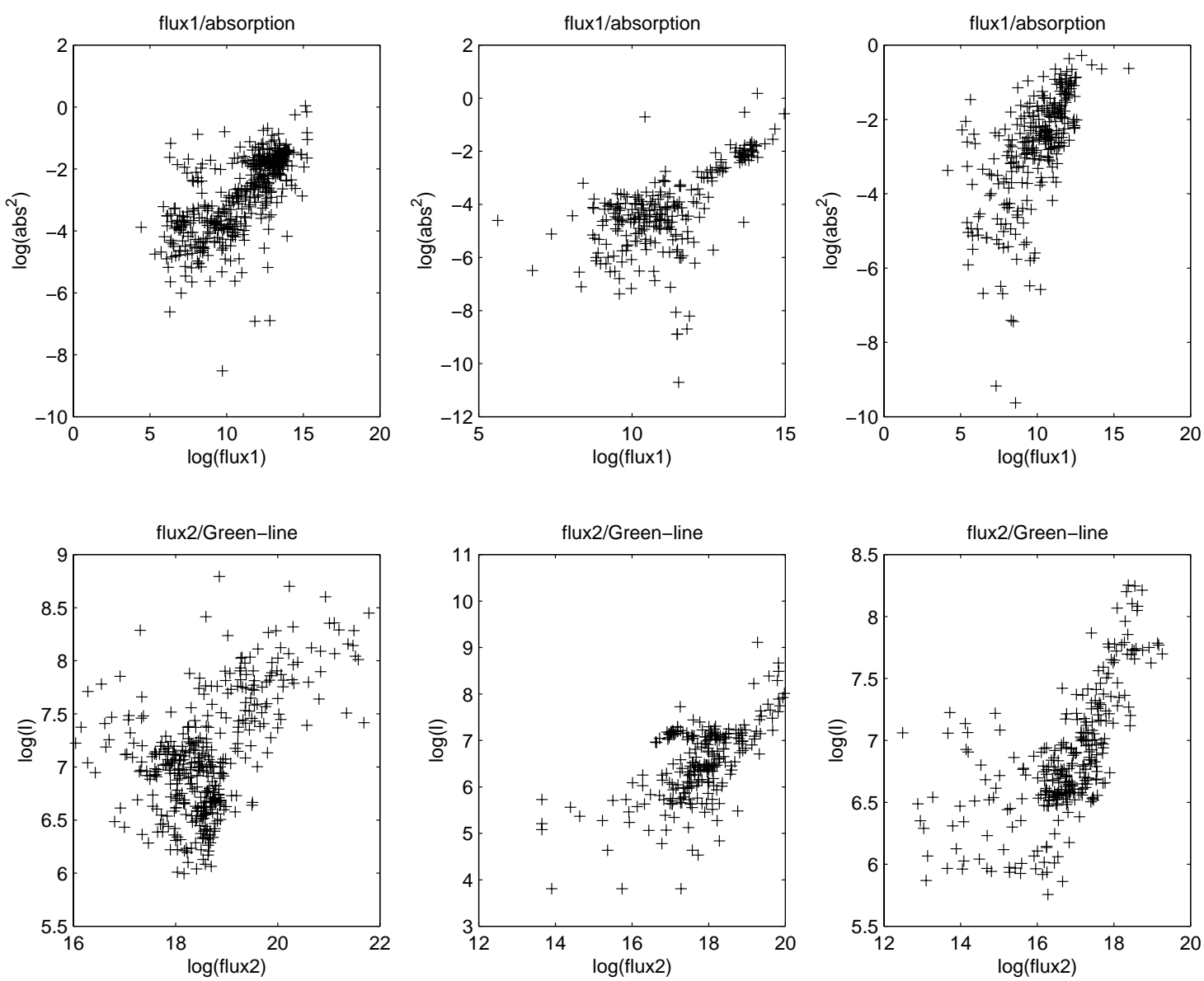

Fig. 3. Dispersion plots for the electron flux and absorption (upper panels) and the electron flux and green-light intensity (bottom panels) for the three data sets at Tromsø: (a) 13-14 Feb 1996, (b) 9 Nov 1998, and (c) 12 Feb 1999. The $y$-axis in the upper panels gives the logarithm of the squared absorption and the logarithm of the green-light intensity in the bottom panels. In all cases, the $x$-axis gives the logarithm of the flux at the corresponding height/energy range.

the logarithm of $\left(\mathrm{Int} / \mathrm{Abs}^{2}\right)$; thus, the dispersion among parameters $a$ and $b-$ and in fact in $E o-$ is not too large, with the worst case being on 9 November 1998. During this period, we observed the brightest aurora, but most of the time it was slightly north of the IRIS field of view. The fact that the linear regressions are essentially the same for different data sets is proof that this technique of estimating $E o$ from Abs and Int can be applied to any IRIS and DASI data set pair without the assistance of EISCAT data.

Figures 5 and 6 display various characteristic energies estimated from different spectral distributions employing the ratio of fluxes $\left(F_{2} / F_{1}\right)$, and those estimates for similar conditions, but making use of the ratio (Int/Abs ${ }^{2}$ ) of the optical intensity to the square of the absorption. We are comparing the flux-averaged energy, $\langle E\rangle$, with the characteristic energies, $E o$, for various spectral distributions between energies $E_{1}$ and $E_{2}$. We consider exponential and mixed distributions of first (exponential/Maxwellian) and second (exponential/power-2) orders. If the flux spectrum were well represented by any of these distributions, then $\langle E\rangle \sim E o$. We are also including the determinations of $E o$, consider- ing full Maxwellian and full power-2 distributions for comparison purposes. In all figures, the top panel corresponds to the 13-14 February 1996 period (a), the middle panel to the 9 November 1998 period (b), and the bottom panel to the 12 February 1999 period (c). As a reference and to facilitate the comparisons, we are including in all figures the flux-averaged energy $\langle E\rangle$ (in black) determined by integrating over the spectrum in the range $1-200 \mathrm{keV}$, for all periods and all times. As mentioned in the introduction, for the exponential and mixed distributions, we have $\langle E\rangle \sim E o$.

Figure 5 also includes the values of Eo estimated from Eq. (2), assuming two exponential distributions, one in height range $98-120 \mathrm{~km}$ (in green, $E_{1}=20 \mathrm{keV}$ and $E_{2}=$ $2 \mathrm{keV}$ ) and the other in height range $89-117 \mathrm{~km}$ (in blue, $E_{1}=40 \mathrm{keV}$ and $E_{2}=3 \mathrm{keV}$ ). The red curve gives the values of $E o$ obtained by applying Eq. (8) for an exponential distribution. The corresponding exponential calculations (the red and blue curves) generally follow each other closely. The generally good correspondence between the black and green curves in all panels in Fig. 5 (and particularly at the top and bottom panels) shows that the flux-averaged energy 

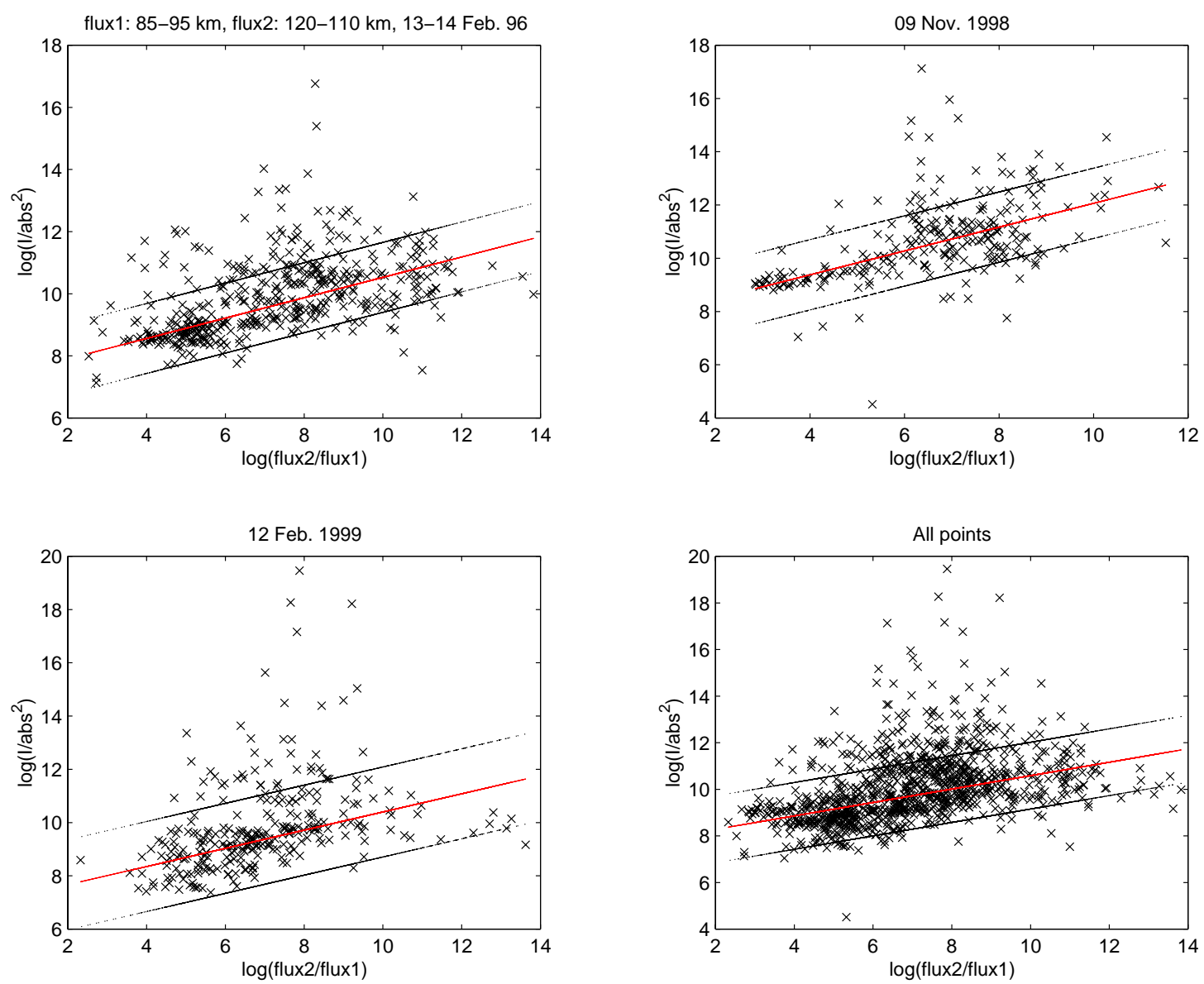

Fig. 4. Dispersion plots between the logarithm of the ratio of the optical intensity to the square of absorption (Int/Abs ${ }^{2}$ ), and the logarithm of the ratio of the corresponding fluxes $\left(F_{2} / F_{1}\right)$, for the same data sets as in Fig. 3 (panels (a), (b), and (c)). Panel (d) displays all the data points together. The regression lines are shown in red while the dotted lines on both sides give the interval at one standard deviation.

$\langle E\rangle$ is well represented by the characteristic energy $E o$ from an exponential spectrum in the $98-120 \mathrm{~km}$ range. This indicates that most of the flux is concentrated at the lower energy side of the spectrum and the spectral shape is closely exponential at these energies. On the other hand, the estimate of $E o$ from an exponential distribution over the full energy range (blue curve) is systematically greater than $\langle E\rangle$, which means that the flux at higher energies is stronger than in the exponential approximation. This is evidence of the presence of acceleration processes and the fact that a Maxwellian or even a modified power-law spectrum may give a better representation of the higher energy fluxes (see Fig. 6a).

Figures $6 \mathrm{a}$ and $\mathrm{b}$ display together the Eo estimates by considering two mixed distributions, an exponential/Maxwellian (blue curve) and an exponential/power-2 (red curve); the magenta and green curves show $E o$ for, respectively, a full Maxwellian distribution and a full power- 2 distribution. Figure 6 a employs the ratio $\left(F_{2} / F_{1}\right)$ at energies $E_{1}=40 \mathrm{keV}$ and $E_{2}=3 \mathrm{keV}$ (actually the fluxes are averaged over the corresponding height ranges $85-95 \mathrm{~km}$ and $108-120 \mathrm{~km}$ ) and Fig. $6 \mathrm{~b}$ data from ratio $\left(\mathrm{Int} / \mathrm{Abs}^{2}\right)$ at the assumed similar height ranges. The black curve in all frames is the flux- averaged energy $\langle E\rangle$. The determinations employing ratio $\left(F_{2} / F_{1}\right)$ are very sensitive to small or noisy values of the higher energy flux $F_{1}$ and, hence, errors can be greatly amplified. This is also true for the ratio $\left(\mathrm{Int} / \mathrm{Abs}^{2}\right)$ for low or noisy values of the absorption. The apparent overflows in Fig. 6b around 19:00 UT and 19:30 UT in period (a), and around 18:20 UT and 18:30 UT in period (b) may be associated with particularly bad values of the absorption (in Fig. 1).

In both Figs. $6 a$ and $b$ and in periods (b) and (c), the estimates of $E o$ from the mixed distributions (blue and red curves) appear to follow $\langle E\rangle$ (black curve) closer than in period (a), and also generally closer than the full Maxwellian and full power-2 estimates (magenta and green curves), particularly for period (c). Eo, from the full Maxwellian and full power-2 distributions, corresponds with mixed distribution estimates but at a much smaller energy "threshold" $E_{2}$. This means that $\langle E\rangle$ could be better represented by lowering the energy threshold $E_{C}$, particularly in period (a). This, however, does not seem to be justified by any physical consideration, and we should conclude that the flux spectrum, at least in this case, could not be represented by a simple analytical distribution. We would have to look systematically 

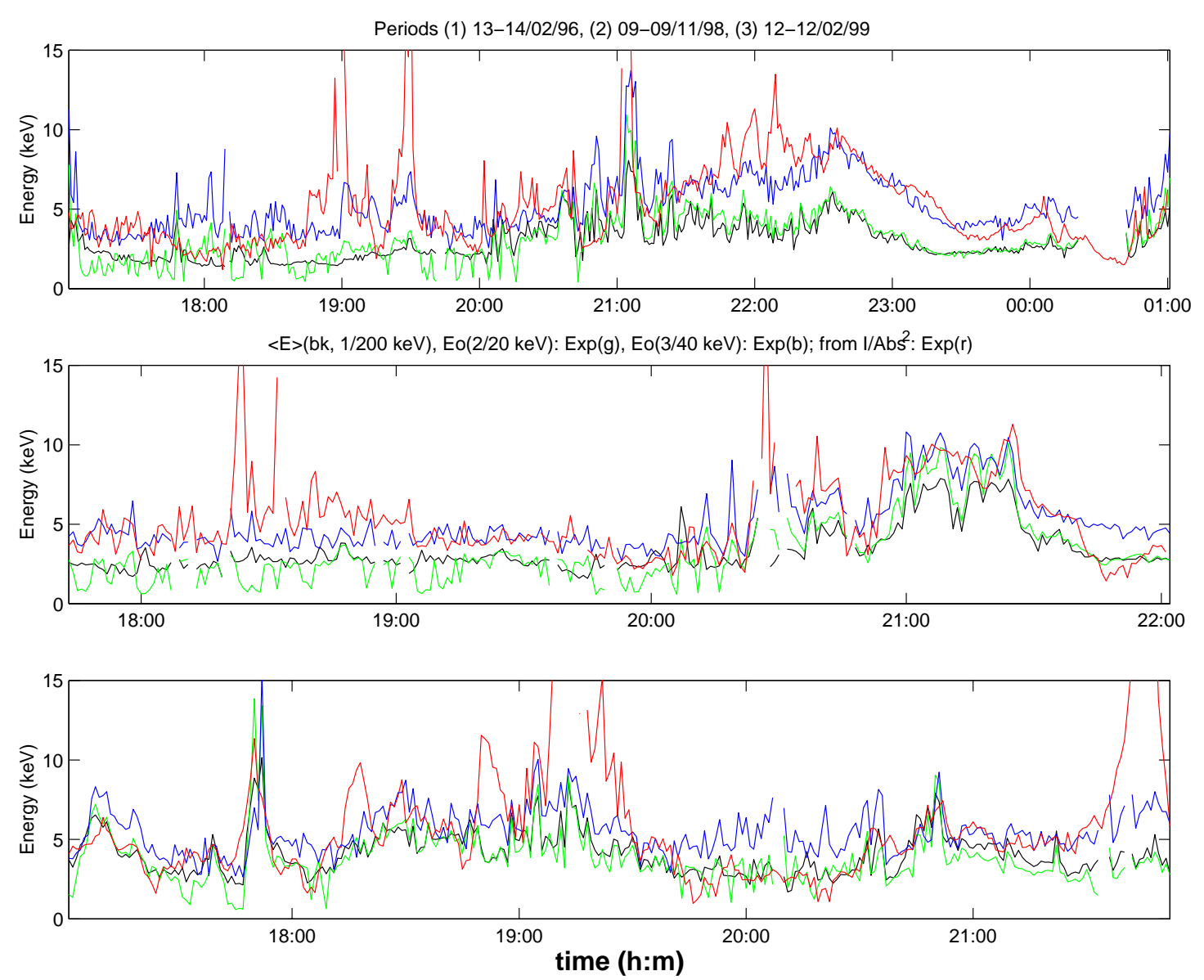

Fig. 5. (Top panel) 13-14 Feb 1996, (middle panel) 9 Nov 1998, and (bottom panel) 12 Feb 1999. In all cases, the black curve shows the flux-averaged energy $\langle E\rangle$ over the full spectrum in the 1-200 keV range. The green and the blue curves give, respectively, the estimated $E o$ from $\log \left(F_{2} / F_{1}\right)$, for two exponential distributions, the first in height range $98-120 \mathrm{~km}\left(E_{1}=20 \mathrm{keV}\right.$ and $\left.E_{2}=2 \mathrm{keV}\right)$ and the second in height range $89-117 \mathrm{~km}\left(E_{1}=40 \mathrm{keV}\right.$ and $\left.E_{2}=3 \mathrm{keV}\right)$. The red curve gives the values of $E o$ from $\log \left(\operatorname{Int} / \mathrm{Abs}^{2}\right)$ for an exponential distribution.

and one by one at all the spectra and determine how close they follow a given distribution in the energy range $\left(E_{1}, E_{2}\right)$.

From the results of the linear regression and Eqs. (7) and (8), parameter $1 / \log \left(\mathrm{Int} / \mathrm{Abs}^{2}\right)$ is, therefore, a good indicator of the characteristic energy over the common field of view of IRIS and DASI, and will allow for the determination of "energy maps" of the electron precipitation evolving in time. We are presenting in Fig. 7 two examples of such energy maps: (a) at 20:42 UT ( 23:00 LT) on 13 February 1996, and (b) at 20:24 UT ( $\sim 22: 45$ LT) on 9 November 1998. We will not go into any detail on the structure of the "energy maps"; we refer to Kosch et al. (2001) for complementary information and discussion. These characteristic energy maps were calculated from the $\log \left(\mathrm{Int} / \mathrm{Abs}^{2}\right)$ in the common field of view by extrapolating the results of the linear regressions (in Fig. 4, and Eq. 8) for a mixed Exponential/Maxwellian distribution. The figure shows in the top panel the absorption map from IRIS and in the middle panel the optical intensity map from DASI. The bottom panel shows the energy map. The colour bars on the right-hand side are given, respectively, in $\mathrm{dB}$ (top) for the absorption, in $\mathrm{kR}$ (middle) for the optical intensity, and in $\mathrm{keV}$ (bottom) for the characteristic energy. Optical signatures of arcs are generally more evenly distributed in space, being narrow and elongated along the L-shells, while the absorption regions appear more structured and patchy, although globally aligned with the arcs.

Figure 7a shows a well-defined, slowly varying quiet-time arc, while the auroral form in Fig. 7b is associated with folding and spiral formation in a disturbed-time arc structure. Absorption regions extend south of the optical signatures which seems to indicate that energetic electrons responsible for the absorption reach deeper into the inner magnetosphere being accelerated and dispersed before precipitation. The time shown in Fig. 7a is also shown in Kosch et al. (2001), but our result employs the correct ratio of the optical intensity to the square of absorption (see Introduction) and it assumes a mixed distribution while theirs considers a full Maxwellian distribution. Our energy levels are smaller than theirs, which is explained partly by their error of a factor of 2 or so, and partly by the different spectral distribution and the smaller 



(a)
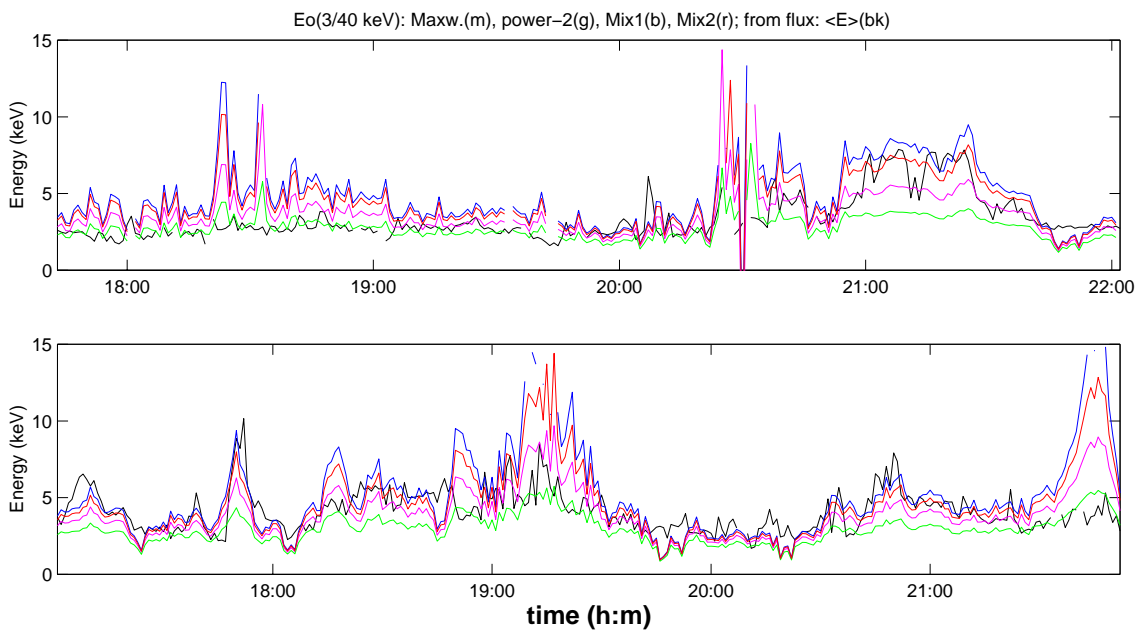

(b)

Fig. 6. (Top panel) 13-14 Feb 1996, (middle panel) 9 Nov 1998, and (bottom panel) 12 Feb 1999. In all cases, the black curve shows the same energy $\langle E\rangle$ as Fig. 5. Both Figs. 6a and b display together the Eo estimates by considering two mixed distributions, an exponential/Maxwellian (blue curve) and an exponential/power-2 (red curve); the magenta and green curves show Eo for, respectively, a full Maxwellian distribution and a full power- 2 distribution. Figure $6 \mathrm{a}$ employs the ratio $\left(F_{2} / F_{1}\right)$ at energies $E_{1}=40 \mathrm{keV}$ and $E_{2}=3 \mathrm{keV}$, and Fig. $6 \mathrm{~b}$ applies data from ratio $\left(\mathrm{Int} / \mathrm{Abs}^{2}\right)$.

average energy difference $\left(E_{1}-E_{2}\right)$ they consider (18 keV while ours is $37 \mathrm{keV}$ ). The structure of the energy maps is otherwise very similar.

\section{Conclusion}

The results and the heuristic arguments presented in Kosch et al. (2001), and in this study, show the presence of a gen- 

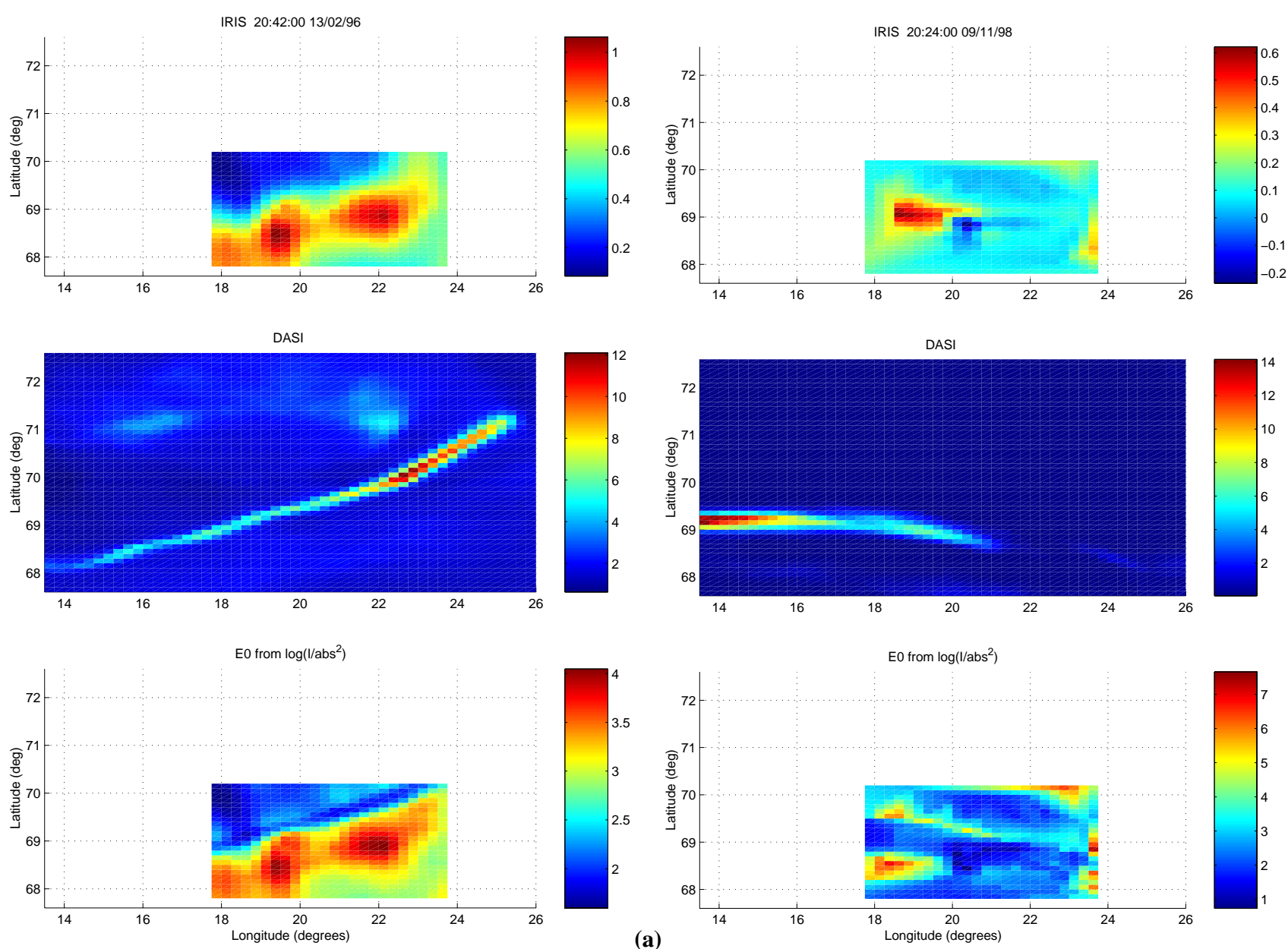

(b)

Fig. 7. Energy maps for auroral arcs as seen by IRIS and DASI in their respective fields of view at (a) 20:42 UT on 13 Feb 1996 and at (b) 20:24 UT on 9 Nov 1998. (Top panels) 2-D absorption distribution, (middle panels) green-light intensity from DASI, and (bottom panels) characteristic energy map in the common field of view of the instruments. The colour bars on the right-hand side are, respectively, in $\mathrm{dB}$ (top), in $\mathrm{kR}$ (middle), and in $\mathrm{keV}$ (bottom).

erally good linear correlation between the particle fluxes and the optical emission and absorption, at the two height ranges where most of the optical emission $(557.7 \mathrm{~nm})$ and the absorption take place. This paper also corrects the derivations and results shown in Kosch et al. (2001); they made a mistake by comparing the characteristic energy $E o$ with $\log \left(\operatorname{Int}^{0.5} / \mathrm{Abs}\right)$. This mistake did indeed propagate to their results, but fortunately because the characteristic energy $E o$ is proportional to $\log \left(\operatorname{Int} / \mathrm{Abs}^{2}\right)$, and not to $\log \left(\mathrm{Int}{ }^{0.5} / \mathrm{Abs}\right)$ as they wrongly stated, the structure of their "energy maps" was not affected but only the energy levels by a linear factor of the order of 2.

Optical intensity and absorption compare very well with average fluxes at $85-95 \mathrm{~km}$ height range (or $30-70 \mathrm{keV}$ energy range) and at $108-120 \mathrm{~km}$ height range (or $2-7 \mathrm{keV}$ energy range), respectively. We have analysed three data sets of simultaneous observations by the EISCAT, IRIS and DASI instruments, in period (a) 13 February 1996, in period (b) 9 November 1998, and in period (c) 12 February 1999. The energy-flux spectrum was calculated from the "inversion" of the EISCAT electron density profiles. The results also show the generally good linear dependence between the logarithm of the ratio of the average fluxes, at the corresponding energy bands, and the logarithm of the ratio of the optical intensity to the absorption measured at Troms $\varnothing$. This makes possible, in principle, to estimate the characteristic energy of the precipitating electrons and to determine the "energy map" of the precipitation over the common field of view of the IRIS and DASI instruments. We have also tried to see if a physical parameter, such as the flux-averaged energy - directly derived from the EISCAT measurements - can be employed both as a reference for, and to further qualify, our determinations of the characteristic energy, thus, helping us to decide which type of spectral distribution (given by Eq. 1 or Eq. 4) may give a better representation of the energy-flux spectrum.

Investigating the individual data sets of Kosch et al. (2001) (13 February 1996 and 9 November 1998), as well as another data set (12 February 1999) shows that the linear regression 
is essentially the same for all cases. Hence, it is possible to use IRIS and DASI data to estimate the characteristic energy $E o$, even when EISCAT radar data is unavailable. We have, on the other hand, only partly succeeded in our attempt to give a more physical basis to the estimation of the characteristic energy $E o$ by trying to define a reference, the flux-averaged energy $\langle E\rangle$, directly derived from the EISCAT measurements, and to justify the choice or one or another type of spectral distribution that may better represent the observations. Our results show that the spectrum of precipitation is closely exponential in the $98-120 \mathrm{~km}$ height range, while at lower heights and higher energies it may follow a Maxwellian or modified power-law distribution. Therefore, a "mixed" distribution, combining an exponential spectrum at the lower energies with a Maxwellian, or even a higher order power-law spectrum at the higher energies, may give a better representation of the characteristic energy and the spectral shape than a single exponential dependence for the full spectrum. Our analysis has underlined the exponential and mixed distributions of first (exponential/Maxwellian) and second (exponential/power-2) orders. A "mixed" distribution should be a function of some "threshold" energy $E_{C}$ defining some transition between narrow and broad pitch-angle scattering, and that may even depend on $E o$, and change with time and the geomagnetic disturbance level. In this paper we have assumed a fixed value of $E_{C}=25 \mathrm{keV}$, considering the fact that up to $20 \mathrm{keV}$ the shape of the flux spectrum could be reasonably represented by an exponential distribution.

Moreover, if the energy-flux spectrum were well represented by any of the distributions in Eq. (4), then the fluxaveraged energy will closely approximate the characteristic energy of the spectrum (thus, $\langle E\rangle \sim E o$ ). For the corresponding "plain" distributions in Eq. (1), $\langle E\rangle$ is two, three times, etc., greater than $E o$. In periods (b) and (c), the estimates of $E o$ from the mixed distributions appear to follow $\langle E\rangle$ closer than in period (a), and also generally closer than the full Maxwellian and full power- 2 estimates, particularly for period (c). $E o$, from the full Maxwellian and full power-2 distributions, corresponds with mixed distribution estimates but at a much smaller energy "threshold" $E_{2}$. This means that $\langle E\rangle$ could be better represented by lowering the energy threshold $E_{C}$, particularly in period (a). This, however, does not seem to be justified by any physical consideration and we should conclude that the flux spectrum, at least in this case, could not be represented by a simple analytical distribution. We leave the task of determining a more precise value (or values) for energy $E_{C}$ to a follow-up study. It may indeed be possible to estimate this energy by looking at all individual spectra and systematically fitting - by a least mean square technique - the "mixed" spectral shapes given by Eq. (4). This kind of analysis will also help to establish the best average spectral shape in energy range $\left(E_{1}, E_{2}\right)$, closely representing the energy-flux spectrum in various conditions.

Acknowledgements. EISCAT is supported by the Suomen Akatemia (Finland), the Centre National de la Recherche Scientifique (France), the Max-Planck Gesellschaft (Germany), the National Institute of Polar Research (Japan), Norges Almenraitenskapelige Forksningrad (Norway), Naturretenskapliga Forskningsradet (Sweden), and the Particle Physis and Astronomy Research Council (UK). We thank Steve Maple at Lancaster University for the help in processing the IRIS data.

The Editor-in-Chief thanks D. Fontaine for his help in evaluating this paper.

\section{References}

Berkey, F. T.: Coordinated measurements of auroral absorption and luminosity using the narrow beam technique, J. Geophys. Res., 73, 319, 1968.

Browne, S., Hargreaves, J. K., and Honary, B.: An imaging riometer for ionospheric studies, Electronics and Communications, 7, 209, 1995.

Collis, P. N., Hargreaves, J. K., and White, G. P.: A localised corotating auroral absorption event observed near noon using imaging riometer and EISCAT, Ann. Geophysicae., 14, 1305, 1996.

del Pozo, C. F., Hargreaves, J. K., and Aylward, A. D.: Ion composition and effective ion recombination rate in the nighttime auroral ionosphere, J.A.T.P., 55, 1919, 1997.

del Pozo, C. F., Turunen, E., and Ulich, T.: Negative ion composition in the auroral mesosphere during a PCA event around sunset, 17, 782, 1999.

Egeland, A. and Omholt, A.: A statistical study of Carl Stormer's height measurements of aurora borealis, in: Aurora and Airglow, (Ed) McCormac, B. M., Reinhold, N.Y., 143-158, 1967.

Gustafsson, G.: Spatial and temporal relations between auroral emission and cosmic noise absorption, Planet. Space Sci., 17, 1961, 1969.

Hargreaves, J. K.: The Solar-Terrestrial Environment, Cambridge University Press, 1992.

Johansen, O. E.: Variations in energy spectrum of auroral electrons detected by simultaneous observation with photometer and riometer, Planet. Space Sci., 13, 225, 1965.

Kosch, M. J., Honary, F., del Pozo, C. F., Marple, S. R., and Hagfors, T.: High resolution maps of the characteristic energy of precipitating auroral particles, J. Geophys. Res., 106, 28 925, 2001.

Kosch, M. J., Hagfors, T., and Nielsen, E.: A new digital all-sky imager experiment for optical auroral studies in conjunction with the Scandinavian twin auroral radar experiment, Rev. Sci. Instruments, 69, 2, 578, 1998.

Oksman, Y.: Aurorae, in Special phenomena of the upper atmosphere, in: The Upper Atmosphere, (Eds) Dieminger, W., Hartmann, G. K., and Leitinger, R., Springer-Verlag, 829-858, 1996.

Rees, M. H.: Auroral ionisation and excitation by incident energetic electrons, Planet. Space Sci., 11, 1209, 1963.

Rees, M. H.: Physics and Chemistry of the Upper Atmosphere, Cambridge University Press, 1989.

Rishbeth, H. and Garriott, O. K.: Introduction to Ionospheric Physics, Academic Press, London, 1969.

Rishbeth, H. and Williams, P. J. S.: The EISCAT ionospheric radar: the system and its early results, Q. J1 R. Astr. Soc., 26 478, 1985.

Rostoker, G., Kamide, Y., and Winningham, J. D.: Energetic particle precipitation into high-latitude ionosphere and the auroral electrojets, 3. Characteristics of electron precipitation into the morning sector auroral oval, J. Geophys. Res., 90, 7495, 1985.

Stoker, P. H., Mathews, M. J., and Scourfield, M. W. J.: Coordinated measurements of auroral light intensities and riometric radiowave absorption, Geophys. Res. Lett., 23, 641, 1996. 